\title{
Nicotinergic Impact on Focal and Non-Focal Neuroplasticity Induced by Non-Invasive Brain Stimulation in Non-Smoking Humans
}

\author{
Nivethida Thirugnanasambandam', Jessica Grundey', Kim Adam', Anne Drees', Angela C Skwirba', \\ Nicolas Lang', Walter Paulus' and Michael A Nitsche*,I \\ 'Department of Clinical Neurophysiology, Georg-August-University Goettingen, Goettingen, Germany; 'Department of Neurology, \\ Christian-Albrechts University, Kiel, Germany
}

\begin{abstract}
Nicotine improves cognitive performance and modulates neuroplasticity in brain networks. The neurophysiological mechanisms underlying nicotine-induced behavioral changes have been sparsely studied, especially in humans. Global cholinergic activation focuses on plasticity in humans. However, the specific contribution of nicotinic receptors to these effects is unclear. Henceforth, we explored the impact of nicotine on non-focal neuroplasticity induced by transcranial direct current stimulation (tDCS) and focal, synapse-specific plasticity induced by paired associative stimulation (PAS) in healthy non-smoking individuals. Forty-eight subjects participated in the study. Each subject received placebo and nicotine patches combined with one of the stimulation protocols to the primary motor cortex in different sessions. Transcranial magnetic stimulation (TMS)-elicited motor-evoked potential (MEP) amplitudes were recorded as a measure of corticospinal excitability until the evening of the second day following the stimulation. Nicotine abolished or reduced both PAS- and tDCS-induced inhibitory neuroplasticity. Non-focal facilitatory plasticity was also abolished, whereas focal facilitatory plasticity was slightly prolonged by nicotine. Thus, nicotinergic influence on facilitatory, but not inhibitory plasticity mimics that of global cholinergic enhancement. Therefore, activating nicotinic receptors has clearly discernable effects from global cholinergic activation. These nicotinegenerated plasticity alterations might be important for the effects of the drug on cognitive function.
\end{abstract}

Neuropsychopharmacology (20II) 36, 879-886; doi:I0.1038/npp.2010.227; published online I5 December 20I0

Keywords: neuroplasticity; nicotine; paired associative stimulation; transcranial direct current stimulation; transcranial magnetic stimulation

\section{INTRODUCTION}

Smoking tobacco is the single largest preventable cause of mortality and morbidity (Peto et al, 1992). Nicotine is the primary constituent of tobacco that is responsible for its addictive properties. Nicotine is the classical agonist at nicotinic acetylcholine receptors, which are ligand-gated cation channels. Studies in animals and human beings have shown that nicotine improves attention as well as working and long-term memory (Froeliger et al, 2009; Hahn and Stolerman, 2002; Kumari et al, 2003). Although many studies focused on the behavioral effects of nicotine in healthy human beings and patients (Jacobsen et al, 2004; Sacco et al, 2005), very few have investigated the

*Correspondence: Professor Dr MA Nitsche, Department of Clinical Neurophysiology, Georg-August-University Goettingen, RobertKoch-Strasse 40, Goettingen 37075, Germany, Tel: + 4955139957 or + 4955 ।39 |263।, Fax: + 4955 139862। or + 4955 1398126 E-mail: mnitsch I@gwdg.de

Received 13 September 20 I0; revised 3 November 20 10; accepted I5 November 2010 nicotinergic impact on cortical excitability and plasticity, which are the likely neurophysiological basis for the cognitive effects of the substance. For global cholinergic enhancement, it was shown that cholinesterase inhibitors reduce intracortical inhibition, increase facilitation, and enhance focal, but diminish non-focal facilitatory plasticity in healthy humans (Korchounov et al, 2005; Kuo et al, 2007). A study on tobacco smokers, who are under chronic nicotine exposure, revealed enhanced motor cortex inhibition and reduced facilitation (Lang et al, 2008). Nicotine also enhances and prolongs the facilitatory after-effects of intermittent theta burst stimulation in human motor cortex (Swayne et al, 2009). Thus, global cholinergic and nicotinergic activation might have at least partially dissimilar effects on cortical excitability.

Transcranial direct current stimulation (tDCS) and paired associative stimulation (PAS) are non-invasive brain stimulation techniques that induce neuroplastic cortical excitability alterations (Nitsche and Paulus, 2000, 2001; Nitsche et al, 2003a; Stefan et al, 2000; Wolters et al, 2003). Both techniques induce NMDA- and calcium-dependent 
changes of cortical excitability (Nitsche et al, 2003b; Stefan et al, 2002; Wolters et al, 2003). tDCS modulates spontaneous neuronal activity and excitability by either depolarizing or hyperpolarizing neurons. Anodal tDCS induces depolarization that enhances neuronal excitability, whereas cathodal tDCS hyperpolarizes neurons, decreasing their excitability levels (Nitsche and Paulus, 2000, 2001; Nitsche et al, 2003a). As tDCS affects all neurons beneath the electrodes, it is thought to induce relatively non-focal plasticity. PAS, on the other hand, induces focal, synapsespecific, timing-dependent, associative neuroplasticity in the targeted neurons. Here an electrical pulse to a mixed peripheral nerve at an intensity that activates somatosensory fibers is followed by a suprathreshold magnetic pulse applied to the corresponding area of the primary motor cortex. Depending on the inter-stimulus interval (ISI), there occurs synchronous or asynchronous activation of somatosensory-motor cortical connections that enhance or reduce excitability, respectively (Stefan et al, 2000).

In this study, we aimed to identify the specific contribution of nicotinic receptors to the cholinergic effect on focal and non-focal neuroplasticity by exploring the effects of nicotine on tDCS- and PAS-generated plasticity in healthy non-smoking human beings to improve our comprehension of the cognition-enhancing and addictive properties of this substance. As in the foregoing studies, the motor cortex was taken as a model system in this single-blinded, placebocontrolled, partial cross-over study because it allows a convenient monitoring of excitability alterations by measuring motor-evoked potential (MEP) amplitudes via transcranial magnetic stimulation (TMS).

\section{MATERIALS AND METHODS}

\section{Subjects}

Forty-eight healthy human volunteers participated in the study. All of them were complete non-smokers, that is, none of them had smoked tobacco for at least 3 years before the study. They did not suffer from any chronic or acute medical illness or any history of neurological/psychiatric diseases, and did not take any chronic or acute medication. This information was obtained by a detailed free personal interview with the subjects. Pregnancy, family history of epilepsy, presence of any metallic implant, or cardiac pacemaker was ruled out. All of them were right-handed according to the Edinburgh handedness inventory (Oldfield, 1971). The selection of subjects was not based on their results from previous plasticity experiments in our laboratory; most of them were naïve to the experimental procedure. Table 1 shows the demographic characteristics of the different groups. All subjects gave written informed consent before participating in the study. The experiments were approved by the local ethics committee and conformed to the Declaration of Helsinki. Allocation of the subjects to the respective experimental conditions as well as order of sessions was randomized.

\section{Assessing Motor Cortex Excitability}

Single TMS pulses were delivered from a Magstim 200 stimulator (Magstim Company, Whitland, Dyfed, UK) at a
Table I Comparison of the Subject Characteristics of the Four Experiments

\begin{tabular}{lcccc}
\hline $\begin{array}{l}\text { Stimulation } \\
\text { parameter }\end{array}$ & $\begin{array}{c}\text { Anodal } \\
\text { tDCS }\end{array}$ & $\begin{array}{c}\text { Cathodal } \\
\text { tDCS }\end{array}$ & $\begin{array}{c}\text { PAS } \\
\mathbf{1 0}\end{array}$ & $\begin{array}{c}\text { PAS } \\
\mathbf{2 5}\end{array}$ \\
\hline $\begin{array}{l}\text { Number of subjects } \\
\text { Number (\%) of females }\end{array}$ & 12 & 12 & 12 & 12 \\
$\begin{array}{l}\text { Age of subjects (mean } \pm \text { s.d.) } \\
\text { in years }\end{array}$ & $24.3 \pm 1.1$ & $26.9 \pm 3.5$ & $25.9 \pm 2.1$ & $24.5 \pm 1.3$ \\
\hline
\end{tabular}

The table lists the age and gender characteristics of the subjects who

participated in the four different categories of the experiment. Also the mean of the absolute baseline MEP amplitude values are shown.

frequency of $0.25 \mathrm{~Hz}$ with a figure-of-eight-shaped coil (diameter of one winding, $70 \mathrm{~mm}$; peak magnetic field, $2.2 \mathrm{~T}$ ). The coil was held tangentially to the scalp at an angle of $45^{\circ}$ to the sagittal plane with the coil handle pointing laterally and posterior. This induced a postero-anterior current flow in the brain at an angle that optimally activates the corticospinal system monosynaptically (Di Lazzaro et al, 1998). MEPs were recorded using a surface electromyogram (EMG) setup. Surface EMG electrodes (Ag-AgCl) were placed over the abductor digiti minimi muscle (ADM) in a belly-tendon montage. Signals were amplified, band-pass filtered $(2 \mathrm{~Hz}-2 \mathrm{KHz})$, digitized $(5 \mathrm{KHz})$, and stored in a laboratory computer for offline analysis using Signal software and CED 1401 (Cambridge Electronic Design, Cambridge, UK). MEPs were elicited using single pulse TMS over the motor cortex representation of the ADM. The position of the coil on the scalp, where the stimulus elicited consistently the largest MEP amplitudes for slightly suprathreshold intensity was marked as the motor 'hotspot'. Stimulus intensity was then adjusted to obtain peak-to-peak MEP amplitudes of approximately $1 \mathrm{mV}$ (SI $1 \mathrm{mV}$ ). This TMS intensity was kept constant throughout the experiment. The mean MEP amplitude was calculated from at least 20 pulses for baseline, and post-intervention excitability monitoring. The change of the mean MEP amplitude over time reflects alterations of motor cortex excitability.

\section{Transcranial Direct Current Stimulation}

Twenty-four subjects participated in the tDCS experiments. tDCS was administered by a battery-driven constant current stimulator (Schneider Electronic, Gleichen, Germany) through rubber electrodes covered by saline-soaked sponges $\left(35 \mathrm{~cm}^{2}\right)$. One electrode was placed over the motor cortex representation of the right ADM as determined by single pulse TMS and the other electrode over the contralateral supraorbital region. All subjects received $1 \mathrm{~mA}$ of either anodal (for $13 \mathrm{~min}$ ) or cathodal stimulation (for $9 \mathrm{~min}$ ), combined with nicotine or placebo medication in different experimental sessions. Therefore, 12 subjects received anodal tDCS with nicotine or placebo patches and the remaining 12 received cathodal tDCS with nicotine or placebo patches. This stimulation intensity and duration (13-min anodal tDCS and 9-min cathodal tDCS) generates after-effects on cortical excitability lasting for approximately 60 min after stimulation (Nitsche and Paulus, 2001; 
Nitsche et al, 2003a). The two consecutive experimental sessions per subject were separated by at least 1-week interval.

\section{Paired Associative Stimulation}

Twenty-four subjects participated in the PAS experiment. Here a peripheral electrical pulse over the right ulnar nerve at wrist level was followed by a TMS pulse over the motor cortex representation of the ADM at ISIs of either 10 (PAS10) or $25 \mathrm{~ms}$ (PAS-25). The peripheral pulse was delivered from a Digitimer D185 multipulse stimulator (Digitimer, Welwyn Garden City, UK) at an intensity of $300 \%$ of the sensory perceptual threshold. The suprathreshold magnetic pulse was delivered from a Magstim 200 stimulator with an intensity that elicited MEP amplitudes of approximately SI $1 \mathrm{mV}$. The paired pulses were repeated 90 times at a frequency of $0.05 \mathrm{~Hz}$. This protocol induces long-lasting excitability changes in the motor cortex depending on the ISI duration. An ISI of $10 \mathrm{~ms}$ induces excitability diminution, whereas an ISI of $25 \mathrm{~ms}$ induces facilitation (Stefan et al, 2000; Wolters et al, 2003). The subjects were instructed to count the number of pulses they received at their wrist throughout the whole stimulation duration to guarantee sufficient attention to the procedure, which has been shown to be crucial to obtain the intended effects (Stefan et al, 2004).

\section{Pharmacological Intervention}

Each subject participated in two sessions in randomized order. Nicotine transdermal patches $\left(30 \mathrm{~cm}^{2}\right)$, each containing nicotine $0.83 \mathrm{mg} / \mathrm{cm}^{2}$ releasing $15 \mathrm{mg}$ over $16 \mathrm{~h}$ or placebo patches were administered to all subjects in combination with one of the stimulation protocols - anodal tDCS, cathodal tDCS, PAS-10 or PAS-25. By this dosage of nicotine, physiologically and behaviorally relevant plasma levels are accomplished (Tønnesen et al, 1991). Subjects received the patch $6 \mathrm{~h}$ before the start of the stimulation. This was the approximate time for the plasma level of nicotine to reach its maximum following application of the patch (Nørregaard et al, 1992). The patch was retained until the end of the last after-measurement of the experiment on the evening of the second day. To counteract possible systemic side effects of nicotine, the subjects were instructed to take $20 \mathrm{mg}$ domperidone, a peripheral-acting dopamine D2-receptor antagonist with antiemetic effects, in case of need.

\section{Course of the Experiment}

The subjects received either a placebo or nicotine patch, which was adhered to the left upper arm and remained there until the end of the last after-measurement on the following evening. They were given $20 \mathrm{mg}$ domperidone and asked to take it orally in case of any side effects. Unpublished results from our group show that domperidone alone does not have any significant effect on motor cortical excitability. After $6 \mathrm{~h}$, subjects were seated comfortably in a reclined position on a dentist's chair with proper arm and head rests and asked to relax completely. The EMG electrodes were placed at the right $\mathrm{ADM}$ as described above. The motor 'hotspot' was determined over the left motor cortex and marked with a water-proof skin marker, and the TMS intensity needed to induce MEP amplitudes of $1 \mathrm{mV}$ (SI $1 \mathrm{mV}$ ) size was determined. Twenty MEPs were recorded at this stimulus intensity and the mean MEP amplitude was calculated as the baseline. One of the stimulation protocols, either tDCS or PAS, was administered. At least 20 MEPs were recorded immediately following the stimulation $(0 \mathrm{~min})$ and at time points of $5,10,15,20,25,30,60,90$, and $120 \mathrm{~min}$. For the nicotine patch sessions, the after-measurements were also conducted in the evening of the stimulation day and in the morning and evening of the day following the plasticity induction procedure (Figure 1). We used a waterproof pen to mark the ADM electrodes and TMS coil positions and ensured that these were positioned over the same spot during the whole course of the experiment.

\section{Data Analysis and Statistics}

The individual means of the 20 MEP amplitudes recorded at each time point were calculated for all subjects. The postintervention mean MEP amplitudes from each subject were then normalized to the respective individual mean baseline MEP amplitude. The normalized mean MEP amplitudes from all subjects were pooled together and the grand average across subjects for each time bin was calculated.

A repeated measures ANOVA was performed on the normalized data using MEP amplitude as the dependent factor, including all time points up to $120 \mathrm{~min}$ after stimulation. Drug (nicotine vs placebo) and time points were included as within-subjects factors. Stimulation (anodal tDCS/cathodal tDCS/PAS-10/PAS-25) served as between-subjects factors. The Mauchly test was performed to test for sphericity, and the Greenhouse-Geisser correction applied when necessary. Conditional on significant results of the ANOVA, we performed post hoc comparisons using Student's $t$-tests (paired, two-tailed, $p<0.05$, not adjusted for multiple comparisons) where we compared

\begin{tabular}{|c|c|c|c|c|}
\hline Medication & & TMS & $\begin{array}{l}\text { Plasticity } \\
\text { induction }\end{array}$ & $\begin{array}{l}\text { Monitoring motor cortex } \\
\text { excitability }\end{array}$ \\
\hline Placebo Patch & Wait 6 hours & BL & PAS/tDCS & $\begin{array}{l}\text { MEPs up to } 120 \text { mins following } \\
\text { intervention }\end{array}$ \\
\hline Nicotine Patch & Wait 6 hours & BL & PAS/tDCS & $\begin{array}{l}\text { MEPs until the next evening } \\
\text { following intervention }\end{array}$ \\
\hline
\end{tabular}

Figure I Illustration of the experimental design. Each subject underwent two experimental sessions. At the beginning of the session, the subjects first received either a placebo or a nicotine patch. After a $6 \mathrm{~h}$ break, baseline (BL) motor-evoked potentials (MEPs) of approximately I $\mathrm{mV}$ amplitude were recorded by single pulse transcranial magnetic stimulation (TMS) over the motor cortical representational area of the abductor digiti minimi muscle (ADM). One of the four stimulation protocols (anodal transcranial direct current stimulation (tDCS)/cathodal tDCS/paired associative stimulation (PAS)-25/PAS-10) was then applied. After-measurements were made immediately following the stimulation and once every 5 min for the first $30 \mathrm{~min}$, then once every 30 for 120 min for both sessions. In addition, MEPs were also recorded the same evening, next morning, and evening for the nicotine patch sessions. 
(i) the mean MEP amplitudes at the time points after plasticity induction $v s$ baseline and (ii) the mean MEP amplitudes following nicotine $v s$ placebo at one time point within a stimulation condition. Moreover, we compared absolute baseline MEP values between the stimulation and drug conditions via Student's $t$-tests to exclude $a$ priori differences. $\chi^{2}$ test was performed to look for significant differences in gender distribution between the groups. For comparison of the age of subjects between the groups, Student's $t$-tests (two-tailed, unpaired, $p<0.05$ ) were performed.

\section{RESULTS}

All subjects tolerated the experimental procedure well. None of them complained of any side effects of either nicotine or the stimulation. Especially the participants did not complain of any sedative effects of the patch. During the experiment, they were completely alert and relaxed. As no systemic side effects of nicotine were perceived and none of the participants needed to take domperidone, the subjects were blinded effectively. Gender distribution did not differ significantly between the various groups tested by $\chi^{2}$ test $(p=0.083)$. There were significant differences in the mean age between some of the groups as tested by Student's unpaired $t$-tests. However, the maximum difference of mean age between groups was 2.65 years. Absolute baseline MEP amplitudes did not differ significantly between groups (Student's $t$-test, two-tailed, paired, $p>0.05$ for all cases) or medication conditions (Student's $t$-test, unpaired, twotailed, $p=0.66$ ).

The ANOVA revealed a significant main effect of the between-subjects factor stimulation $(\mathrm{F}(3,44)=18.137$; $p<0.001$ ), in accordance with different effects of inhibitory and facilitatory tDCS and PAS on MEP amplitudes. The main effects of either nicotine $(\mathrm{F}(1,44)=0.093$; $p=0.762)$ or time $(\mathrm{F}(10,440)=1.654 ; p=0.089)$ were not significant. However, the interactions between nicotine $\times$ stimulation $(\mathrm{F}(3,44)=5.498 ; p=0.003)$; time $\times$ stimulation $(\mathrm{F}(30,440)=3.070 ; p<0.001)$ were significant, showing that nicotine had different effects on the above-mentioned stimulation protocols, and that the time course of the effects of these stimulation protocols was not identical. The three-way interaction nicotine $\times$ time $\times$ stimulation $(\mathrm{F}(30,440)=1.848 ; p=0.005)$ was also significant. Thus, application of nicotine patch significantly influenced the after-effects of the different stimulation protocols differently over time.

\section{Nicotinergic Impact on tDCS-Induced Plasticity}

As shown by the post hoc $t$-tests, in the control condition without nicotine, MEPs were significantly enhanced following anodal tDCS for up to $90 \mathrm{~min}$. Cathodal tDCS diminished excitability levels significantly also for up to 90 min after stimulation. Under nicotine, both anodal and cathodal tDCS-induced after-effects were abolished. The post hoc test revealed that for anodal tDCS, post-tDCS MEP amplitudes under nicotine were not different from baseline values, but differed significantly from those under placebo medication (Supplementary Table S1). A trendwise reversal of the effects of anodal tDCS from facilitation to inhibition under nicotine did not reach statistical significance $(p>0.073)$. The excitability diminution induced by cathodal tDCS, as compared with baseline excitability, was also abolished under nicotine; however, relative to the placebo medication condition, nicotine induced only a trendwise change (Figure 2a and b, Supplementary Table S1).

\section{Effect of Nicotine on PAS-Induced Plasticity}

As shown by the post hoc tests, PAS induced a significant facilitation of MEP amplitudes following PAS-25 and excitability diminution following PAS-10, lasting for up to 90 or $120 \mathrm{~min}$ after stimulation (Supplementary Table S1) under placebo medication. Under nicotine, the facilitatory effects of PAS-25 remained significant as compared with the respective baseline MEP for up to $90 \mathrm{~min}$ after PAS. MEP
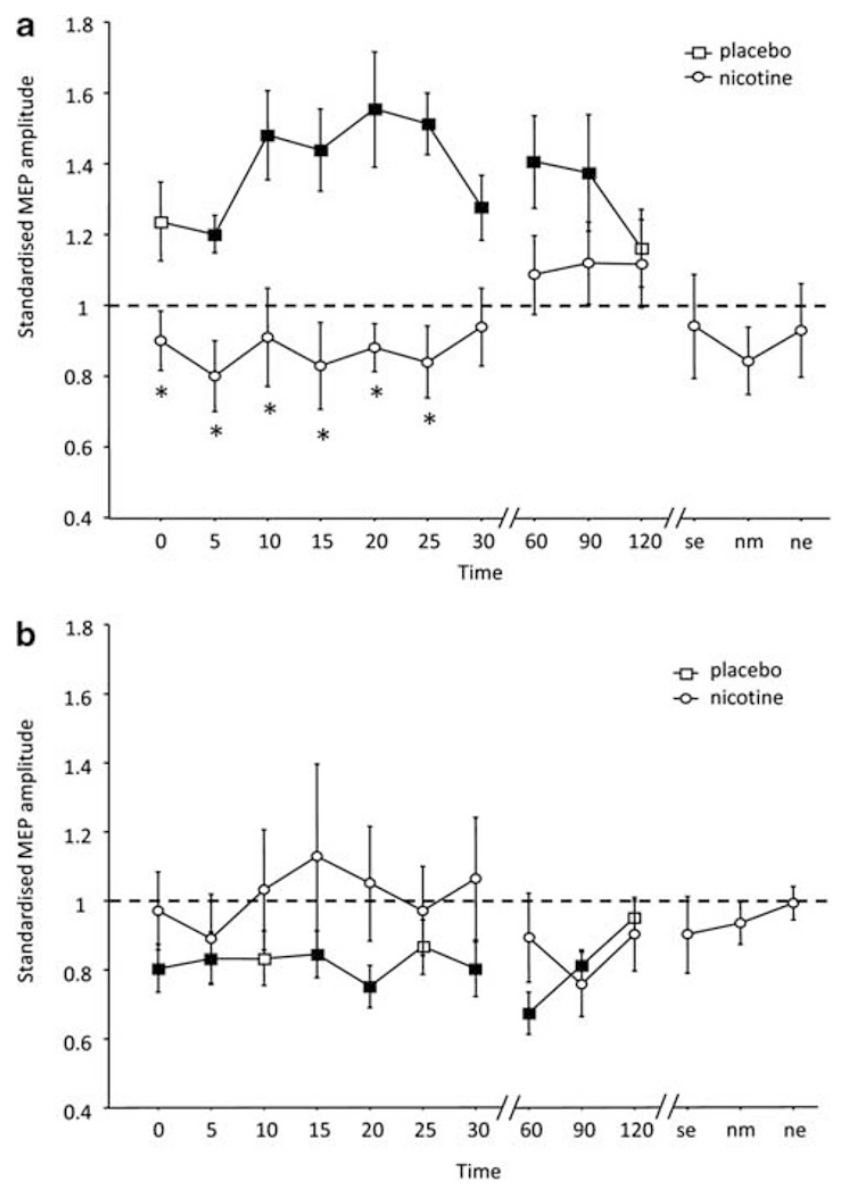

Figure 2 Nicotinergic impact on transcranial direct current stimulation (tDCS)-induced neuroplasticity. Shown are the graphs with motor-evoked potential (MEP) amplitudes standardized to baseline on the $Y$ axis plotted against various time points following the stimulation. (a) In the placebo condition (squares), anodal tDCS enhances motor cortex excitability until $90 \mathrm{~min}$ following stimulation. This effect of anodal tDCS is abolished in the nicotine condition (circles). (b) The cathodal tDCS-induced excitability diminution observed in the placebo condition (squares) is also abolished by application of nicotine (circles). Filled symbols indicate statistically significant deviations from baseline and asterisks indicate significant differences between the control and nicotine conditions (Student's $t$-test, paired, two-tailed, $p<0.05)$. SE, same evening; NM, next morning; NE, next evening. Error bars indicate standard error of mean. 
amplitudes under nicotine did not differ $v s$ the respective placebo medication condition for up to $90 \mathrm{~min}$ after PAS-25, but were significantly larger as compared with placebo $120 \mathrm{~min}$ after PAS-25, being in favor for a prolonged excitability enhancement accomplished by PAS-25 under nicotine. The missing difference of PAS-25 under nicotine relative to baseline excitability $120 \mathrm{~min}$ after plasticity induction might be caused by the relatively large variability of the MEPs at this time point (see Supplementary Table S1). The inhibitory effect of PAS-10 was abolished under nicotine. Consequently, the post hoc tests revealed no significant differences of the respective MEP amplitudes relative to baseline, but significant differences of the respective MEP amplitudes relative to those under placebo medication (Figure $3 a$ and $b$ ).
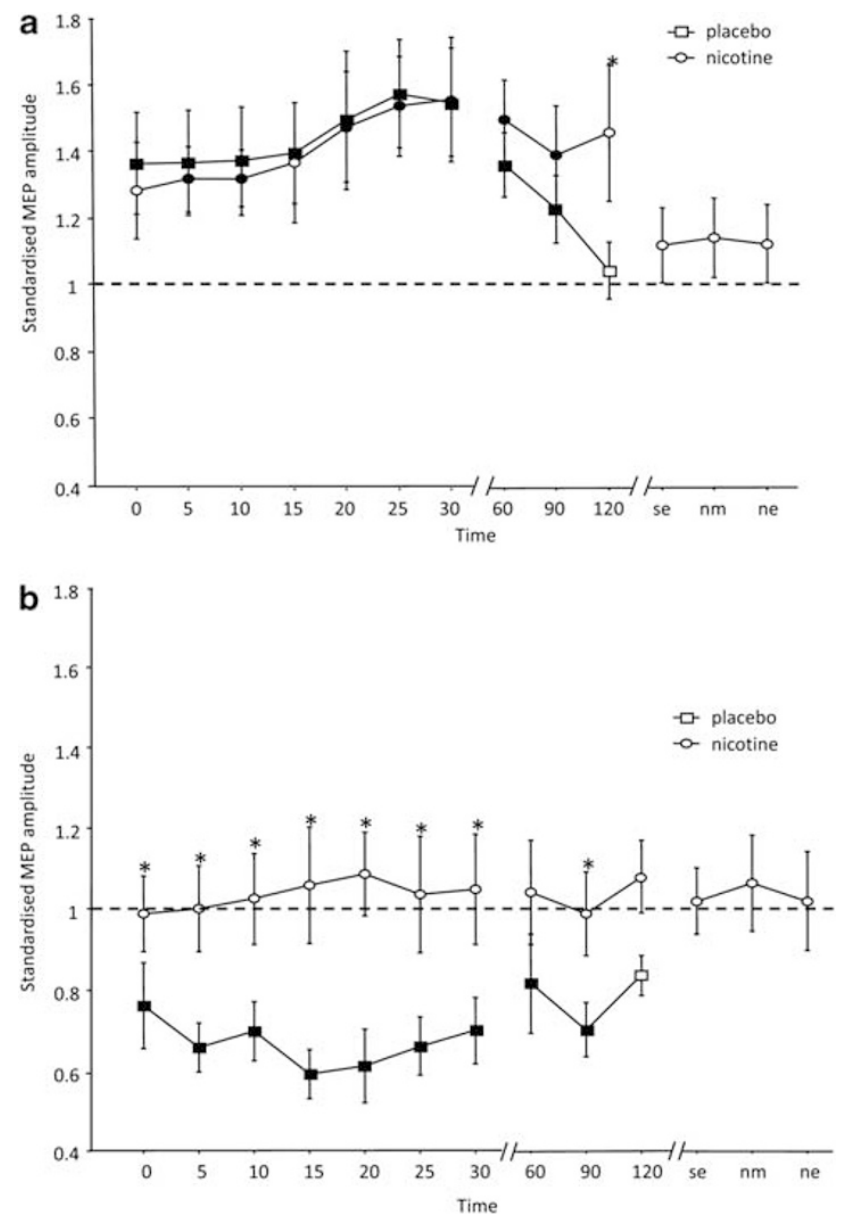

Figure 3 Nicotinergic impact on paired associative stimulation (PAS)induced neuroplasticity. The graphs show baseline-standardized motorevoked potential (MEP) amplitudes on the $Y$ axis plotted at different time points following the stimulation. (a) In the placebo condition (squares), PAS-25 induced a clear increase of MEP amplitudes lasting for 90 min after stimulation. With nicotine (circles), the facilitatory after-effect of PAS-25 is slightly prolonged. (b) PAS- 10 under placebo medication (squares) induced a prominent decrease of MEP amplitudes significantly until 90 min after stimulation. Nicotine (circles) abolished the inhibitory after-effect of PAS10. Filled symbols indicate statistically significant deviations from baseline and asterisks indicate significant differences between the control and nicotine conditions (Student's t-test, paired, two-tailed, $p<0.05$ ). SE, same evening; NM, next morning; NE, next evening. Error bars indicate standard error of mean.

\section{DISCUSSION}

This study shows that in healthy non-smoking individuals nicotine prominently affects neuroplasticity. Our data illustrate that (i) nicotine exposure slightly prolongs or at least preserves the synapse-specific cortical excitability enhancement induced by PAS-25, but abolishes the PAS10 -induced depression of cortical excitability and (ii) the non-focal excitability-enhancing after-effect of anodal tDCS and the excitability diminution caused by cathodal tDCS are both abolished (Figure 4). There is also a trendwise reversal of anodal tDCS-induced facilitation to inhibition under nicotine. Hence, we observe a focusing effect of nicotine on facilitatory neuroplasticity and an abolishment of inhibitory plasticity, the latter irrespective of the focality of stimulation.

Some of our observations match with those of previous studies. The enhancement or preservation of facilitatory plasticity induced by PAS-25 by nicotine is similar to the effect it had on intermittent theta burst stimulation (Swayne et al, 2009). The effect of nicotine on facilitatory plasticity is also comparable with the effect of the cholinesterase inhibitor rivastigmine on identical plasticity induction protocols; however, its effects on inhibitory plasticity are at variance to those under global cholinergic enhancement under rivastigmine (Kuo et al, 2007).

\section{Proposed Mechanism of Action}

The focusing effect of nicotine on facilitatory plasticity, that is, consolidating/preserving focal, PAS-induced, but diminishing non-focal tDCS-generated plasticity, is quite similar to that of global cholinergic enhancement via rivastigmine described in a previous study (Kuo et al, 2007). A likely explanation for this effect is the different impact of

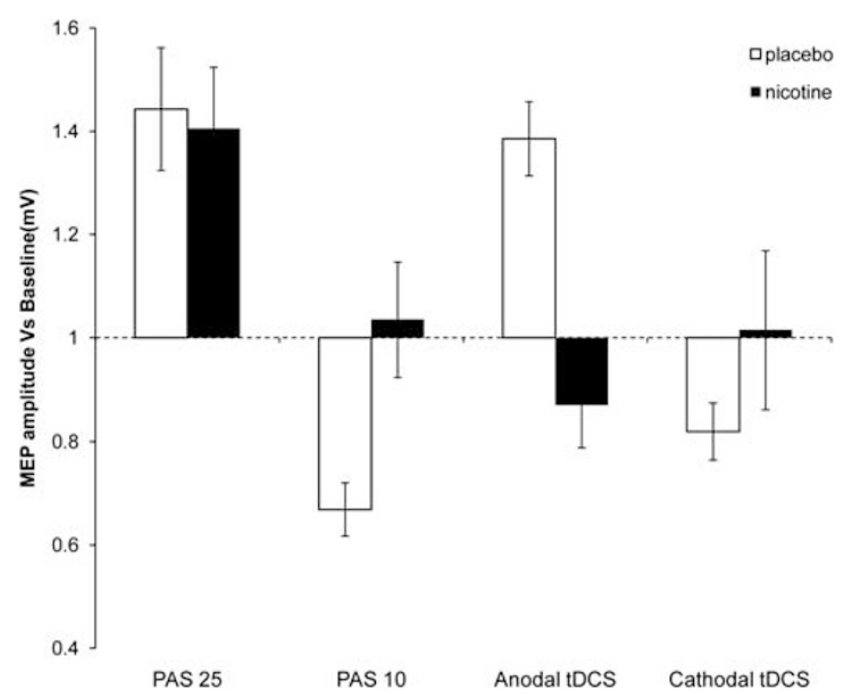

Figure 4 Effect of nicotine on focal/non-focal neuroplasticity induced in the human motor cortex until 30 min following stimulation. Nicotine shows a focusing effect on facilitatory plasticity. Focal facilitatory plasticity is prolonged, whereas non-focal facilitatory plasticity is abolished under nicotine. Nicotine also abolishes any effect of excitability-reducing plasticitygenerating protocols. Each bar represents the mean motor-evoked potential (MEP) amplitude until 30 min after stimulation standardized to the baseline. Error bars indicate standard error of mean. 
cholinergic activation on recurrent activation of afferent input to cortical neurons. It has been shown that excitatory glutamatergic synaptic transmission is suppressed by presynaptic inhibition at intrinsic, recurrent synapses, but not at afferent fiber synapses via cholinergic activation (Hasselmo and Bower, 1992; Hasselmo et al, 1995; Vogt and Regehr, 2001). As PAS induces plasticity by a combination of afferent somatosensory input with a motor cortical stimulus, while tDCS is thought to affect primarily excitability of cortical interneurons (Nitsche et al, 2005), it might be speculated that these specific aspects of the stimulation techniques caused the differences of the effects also in these experiments. In accordance, in animal experiments an inhibitory effect of the activation of nicotinergic subreceptors on feedforward interneurons has has been shown to prevent LTP induction by inhibiting pyramidal neurons, whereas spike-timing-dependent LTP was enhanced (Røsza et al, 2008; Yamazaki et al, 2005), although the latter effect was not shown in all studies (Couey et al, 2007). Owing to the results of this experiment, this effect of cholinergic activation on facilitatory plasticity in human beings can likely be attributed to an impact of nicotinic receptors. Another possible explanation of the results is based on the enhancement of intracellular calcium concentration by nicotinic receptors, for example, the alpha-7 subreceptor. Intracellular calcium is a key determinant of plasticity induction, and the after-effects of tDCS and PAS are calcium dependent (Nitsche et al, 2003b; Stefan et al, 2002). The amount of intracellular calcium determines if inhibitory, facilitatory, or no plasticity is induced. A slight enhancement of intracellular calcium induces long-term depression (LTD), a large enhancement LTP (Lisman, 2001). Medium and very large concentrations of intracellular calcium induce no or convert plasticity, the latter possibly owing to an activation of hyperpolarizing calcium channels (Misonou et al, 2004). As tDCS induces facilitatory plasticity by tonic depolarization of neurons for some minutes, which might activate voltage-dependent calcium channels, whereas PAS is a phasic stimulation technique, which induces only short-lasting depolarization, the amount of intracellular calcium increase caused by tDCS might be larger than that caused by PAS. Therefore, a further calcium enhancement via nicotinic receptor activation might enhance the calcium level above the concentration-inducing LTP-like plasticity, and therefore result in an abolishment of plasticity in case of tDCS, but not PAS. This effect on different kinds of facilitatory plasticity of nicotine might enhance the signal-to-noise ratio (Hasselmo and Barkai, 1995), which would facilitate the representation of meaningful, synchronous inputs and suppress non-meaningful inputs. Indeed, it has been recently suggested that nicotine improves memory performance via calcium-dependent mechanisms in animals (Biała and Kruk, 2009).

In contrast, nicotine abolished all kinds of inhibitory plasticity irrespective of the specific stimulation protocol. One possible explanation might be that the calciumenhancing properties of nicotinic receptor activation here resulted in an intracellular calcium level too large to induce LTD-like plasticity. This mechanism of action would also explain the different effect of global cholinergic activation by rivastigmine on inhibitory plasticity, as described in a previous study, in which this substance prolonged
tDCS- and PAS-generated inhibitory plasticity (Kuo et al, 2007), because muscarinic receptors inhibit voltage-gated calcium channels (Brown, 2010), and thus might counteract the effects of nicotinic receptors in this case. The effects of nicotine on inhibitory plasticity obtained in this study are not in accordance with some animal experiments, where nicotinic activation has been shown to be important for LTD induction (Partridge et al, 2002; Fujii and Sumikawa, 2001); however, the effect of nicotine on LTD seems to depend on the general proneness of the system to inhibitory plasticity (Alzoubi et al, 2007, 2008), which likely differs between animal preparations and in vivo studies in human beings.

It should be mentioned that these mechanistic explanations of the results are hypothetical presently. Alternative explanations, such as the modification of NMDA receptordependent plasticity by nicotine-dependent alteration of GABAergic activity (Couey et al, 2007), cannot be ruled out. These hypotheses should be tested more directly in future studies, for example, by altering the activity of nicotinic subreceptors, or calcium channels, in combination with nicotine exposure.

\section{General Remarks}

The results of this study show that nicotine clearly influences neuroplasticity in non-smoking individuals. Nicotine focuses facilitatory plasticity, whereas it abolishes inhibitory plasticity. The effects differ from those of nonspecific cholinergic activation. The focusing effect of nicotine on facilitatory plasticity might help to explain how this drug improves attention, working memory, and longterm memory in animals and human beings via enhancing the signal-to-noise ratio of plasticity. Also the abolition of inhibitory plasticity by nicotine might affect cognitive processes. First, it might shift the net balance of plasticity more into the direction of facilitatory plasticity, and therefore indirectly enhance cognitive performance further. Second, inhibitory plasticity, especially LTD, has been shown to be directly involved in certain forms of learning and memory formation. Collingridge and co-workers (2010) describe a role of LTD in hippocampal-based learning and memory formation, and recognition memory in perirhinal cortex. As our findings show that nicotine abolishes LTDlike plasticity, it could be speculated that nicotine might worsen LTD-dependent forms of learning and memory. However, an impairment of cognitive functions by nicotine has been rarely described (Toledano et al, 2010), thus further behavioral studies are needed to explore the cognitive effects of nicotine more systematically.

Some limiting aspects of this study should be mentioned. Blinding could have been somewhat compromised considering the fact that the experimenters were not blinded to the intervention. However, the data were collected by more than one investigator without notable difference in the results, which probably indicates low experimenter bias, and experimenters were not informed about hypotheses about expected outcomes of the experiments, which should have limited expectancy effects. Although the subjects did not complain of any sedation owing to nicotine, the degree of alertness was not explicitly assessed and hence its effect on the measurements cannot be ruled out completely. 
We did not measure plasma concentrations of nicotine, thus it could be argued that inter-individual differences of the bioavailability of the substance had an impact on the results. However, as we studied a fairly homogenous group of participants, and we induced plasticity during steadystate drug concentration, we do not think that variability of plasma concentration of nicotine can explain the results. We studied the effect of only a single dosage of nicotine in these experiments. Thus, it cannot be ruled out that the effect of nicotine on plasticity differs dose-dependently, as shown for other neuromodulators, like dopamine (MonteSilva et al, 2009; Monte-Silva et al, 2010). Moreover, as nicotine receptors are rapidly modified by chronic exposure, this study cannot discern between primary effects of nicotine on nicotinic receptors and secondary effects caused by receptor desensitization or upregulation. Moreover, it should not be taken for granted that the effects obtained on motor cortex plasticity, as in this study, translate exactly to other cortices, where nicotinic receptor density, and subreceptor composition might differ (McGehee and Role, 1995; Gotti et al, 2009) Furthermore, the results of a single dosage nicotine application in non-smokers, as performed here, might differ from the effects of nicotine in smokers who are chronically exposed to it. Future studies should address these aspects in larger detail.

\section{CONCLUSION}

The results of this study deliver clear evidence for an important role of nicotine in the formation of neuroplasticity, the likely basis of learning and memory formation, in human beings. Via its focusing effect on facilitatory plasticity, nicotine might be an attractive candidate to enhance these processes in neuropsychiatric diseases accompanied by cognitive decline. The abolition of inhibitory plasticity by nicotine also could have a significant impact on some forms of learning and memory, and also affect addictive behavior to some extent. Moreover, its effect on plasticity might be an important mechanism for starting nicotine consumption, addiction, and the high probability of relapse in smokers. Interestingly, the effects of nicotine on plasticity share some of those of dopamine (Kuo et al, 2008), which might be an explanation for the frequent nicotine consumption in schizophrenia, in which dopaminergic malfunctioning is an important pathological mechanism. Clearly, more studies are needed to explore the exact role of nicotine in healthy human beings and in those suffering from neuropsychiatric diseases to a larger degree. Moreover, the results of this study are important in another aspect. Non-invasive brain stimulation techniques are increasingly used as scientific and therapeutic tools. The results of this study show that the activity of the nicotinergic system might critically affect the effects of brain stimulation. This potentially important confounding factor should thus be taken into account in future studies using brain stimulation.

\section{DISCLOSURE}

The authors declare that, except for income received from my primary employer, no financial support or compensation has been received from any individual or corporate entity over the past 3 years for research or professional service and there are no personal financial holdings that could be perceived as constituting a potential conflict of interest.

\section{ACKNOWLEDGEMENTS}

The study was supported by the Deutsche Forschungsgemeinschaft (DFG Grant NI683/4-1 'Towards risk prediction of nicotine dependency by exploring individual limits of cortical neuroplasticity in humans'; NI 683/4-2 'Impact of the nicotinergic alpha7 receptor on cortical plasticity in smokers and nonsmokers') within the DFG priority program 'Nicotine: Molecular and Physiological Effects in Central Nervous System'.

\section{REFERENCES}

Alzoubi KH, Aleisa AM, Alkadhi KA (2007). Adult-onset hypothyroidism facilitates and enhances LTD: reversal by chronic nicotine treatment. Neurobiol Dis 26: 264-272.

Alzoubi KH, Aleisa AM, Alkadhi KA (2008). Effect of chronic stress or nicotine on hypothyroidism-induced enhancement of LTD: electrophysiological and molecular studies. Neurobiol Dis 32: $81-87$.

Biała G, Kruk M (2009). Influence of bupropion and calcium channel antagonists on the nicotine-induced memory-related response of mice in the elevated plus maze. Pharmacol Rep 61: 236-244.

Brown DA (2010). Muscarinic acetylcholine receptors (mAChRs) in the nervous system: some functions and mechanisms. $J \mathrm{Mol}$ Neurosci 41: 340-346.

Collingridge GL, Peineau S, Howland JG, Wang WT (2010). Longterm depression in the CNS. Nat Rev Neurosci 11: 459-473.

Couey JJ, Meredith RM, Spijker S, Poorthuis RB, Smit AB, Brussaard $\mathrm{AB}$ et al (2007). Distributed network actions by nicotine increase the threshold for spike-timing-dependent plasticity in prefrontal cortex. Neuron 54: 73-87.

Di Lazzaro V, Oliviero A, Profice P, Saturno E, Pilato F, Insola A et al (1998). Comparison of descending volleys evoked by transcranial magnetic and electric stimulation in conscious humans. Electroencephalogr Clin Neurophysiol 109: 397-401.

Froeliger B, Gilbert DG, McClernon FJ (2009). Effects of nicotine on novelty detection and memory recognition performance: double-blind, placebo-controlled studies of smokers and nonsmokers. Psychopharmacology (Berl) 205: 625-633.

Fujii S, Sumikawa K (2001). Nicotine accelerates reversal of longterm potentiation and enhances long-term depression in the rat hippocampal CA1 region. Brain Res 894: 340-346.

Gotti C, Clementi F, Fornari A, Gaimarri A, Guiducci S, Manfredi I et al (2009). Structural and functional diversity of native brain neuronal nicotinic receptors. Biochem Pharmacol 78: 703-711.

Hahn B, Stolerman IP (2002). Nicotine-induced attentional enhancement in rats: effects of chronic exposure to nicotine. Neuropsychopharmacology 27: 712-722.

Hasselmo ME, Barkai E (1995). Cholinergic modulation of activity dependent synaptic plasticity in the piriform cortex and associative memory function in a network biophysical simulation. J Neurosci 15: 6592-6604.

Hasselmo ME, Bower JM (1992). Cholinergic suppression specific to intrinsic not afferent fiber synapses in rat piriform (olfactory) cortex. J Neurophysiol 67: 1222-1229.

Hasselmo ME, Schnell E, Barkai E (1995). Dynamics of learning and recall at excitatory recurrent synapses and cholinergic 
modulation in rat hippocampal region CA3. J Neurosci 15: 5249-5262.

Jacobsen LK, D'Souza DC, Mencl WE, Pugh KR, Skudlarski P, Krystal JH (2004). Nicotine effects on brain function and functional connectivity in schizophrenia. Biol Psychiatry 55: 850-858.

Korchounov A, Ilic TV, Schwinge T, Ziemann U (2005). Modification of motor cortical excitability by an acetylcholinesterase inhibitor. Exp Brain Res 164: 399-405.

Kumari V, Gray JA, ffytche DH, Mitterschiffthaler MT, Das M, Zachariah E et al (2003). Cognitive effects of nicotine in humans: an fMRI study. Neuroimage 19: 1002-1013.

Kuo MF, Grosch J, Fregni F, Paulus W, Nitsche MA (2007). Focusing effect of acetylcholine on neuroplasticity in the human motor cortex. J Neurosci 27: 14442-14447.

Kuo MF, Paulus W, Nitsche MA (2008). Boosting focally-induced brain plasticity by dopamine. Cereb Cortex 18: 648-651.

Lang N, Hasan A, Sueske E, Paulus W, Nitsche MA (2008). Cortical hypoexcitability in chronic smokers? A transcranial magnetic stimulation study. Neuropsychopharmacology 33: 2517-2523.

Lisman JE (2001). Three $\mathrm{Ca}^{2+}$ levels affect plasticity differently: the LTP zone, the LTD zone and no man's land. J Physiol 532 (Part 2): 285.

McGehee DS, Role LW (1995). Physiological diversity of nicotinic acetylcholine receptors expressed by vertebrate neurons. Annu Rev Physiol 57: 521-546.

Misonou H, Mohapatra DP, Park EW, Leung V, Zhen D, Misonou K et al (2004). Regulation of ion channel localization and phosphorylation by neuronal activity. Nat Neurosci 7: 711-718.

Monte-Silva K, Kuo MF, Thirugnanasambandam N, Liebetanz D, Paulus W, Nitsche MA (2009). Dose-dependent inverted Ushaped effect of dopamine (D2-like) receptor activation on focal and nonfocal plasticity in humans. J Neurosci 29: 6124-6131.

Monte-Silva K, Liebetanz D, Grundey J, Paulus W, Nitsche MA (2010). Dosage-dependent non-linear effect of L-dopa on human motor cortex plasticity. J Physiol 588: 3415-3424.

Nitsche MA, Paulus W (2000). Excitability changes induced in the human motor cortex by weak transcranial direct current stimulation. J Physiol 527(Part 3): 633-639.

Nitsche MA, Paulus W (2001). Sustained excitability elevations induced by transcranial DC motor cortex stimulation in humans. Neurology 57: 1899-1901.

Nitsche MA, Fricke K, Henschke U, Schlitterlau A, Liebetanz D, Lang $\mathrm{N}$ et al (2003a). Pharmacological modulation of cortical excitability shifts induced by transcranial direct current stimulation in humans. J Physiol 553(Part 1): 293-301.

Nitsche MA, Nitsche MS, Klein CC, Tergau F, Rothwell JC, Paulus W (2003b). Level of action of cathodal DC polarisation induced inhibition of the human motor cortex. Clin Neurophysiol 114: 600-604.

Nitsche MA, Seeber A, Frommann K, Klein CC, Rochford C, Nitsche MS et al (2005). Modulating parameters of excitability during and after transcranial direct current stimulation of the human motor cortex. J Physiol 568(Part 1): 291-303.

Nørregaard J, Tønnesen P, Simonsen K, Säwe U (1992). Long-term nicotine substitution after application of a 16-h nicotine patch in smoking cessation. Eur J Clin Pharmacol 43: 57-60.

Oldfield RC (1971). The assessment and analysis of handedness: the Edinburgh inventory. Neuropsychologia 9: 97-113.

Partridge JG, Apparsundaram S, Gerhardt GA, Ronesi J, Lovinger DM (2002). Nicotinic acetylcholine receptors interact with dopamine in induction of striatal long-term depression. J Neurosci 22: 2541-2549.

Peto R, Lopez AD, Boreham J, Thun M, Heath Jr C (1992). Mortality from tobacco in developed countries: indirect estimation from national vital statistics. Lancet 339: 1268-1278.

Rózsa B, Katona G, Kaszás A, Szipöcs R, Vizi ES (2008). Dendritic nicotinic receptors modulate backpropagating action potentials and long-term plasticity of interneurons. Eur J Neurosci 27: 364-377.

Sacco KA, Termine A, Seyal A, Dudas MM, Vessicchio JC, Krishnan-Sarin S et al (2005). Effects of cigarette smoking on spatial working memory and attentional deficits in schizophrenia: involvement of nicotinic receptor mechanisms. Arch Gen Psychiatry 62: 649-659.

Stefan K, Kunesch E, Benecke R, Cohen LG, Classen J (2002). Mechanisms of enhancement of human motor cortex excitability induced by interventional paired associative stimulation. J Physiol 543(Part 2): 699-708.

Stefan K, Kunesch E, Cohen LG, Benecke R, Classen J (2000). Induction of plasticity in the human motor cortex by paired associative stimulation. Brain 123(Part 3): 572-584.

Stefan K, Wycislo M, Classen J (2004). Modulation of associative human motor cortical plasticity by attention. J Neurophysiol 92: $66-72$.

Swayne OB, Teo JT, Greenwood RJ, Rothwell JC (2009). The facilitatory effects of intermittent theta burst stimulation on corticospinal excitability are enhanced by nicotine. Clin Neurophysiol 120: 1610-1615.

Toledano A, Alvarez MI, Toledano-Díaz A (2010). Diversity and variability of the effects of nicotine on different cortical regions of the brain-therapeutic and toxicological implications. Cent Nerv Syst Agents Med Chem 10: 180-206.

Tønnesen P, Nørregaard J, Simonsen K, Säwe U (1991). A doubleblind trial of a 16-h transdermal nicotine patch in smoking cessation. $N$ Engl J Med 325: 311-315.

Vogt KE, Regehr WG (2001). Cholinergic modulation of excitatory synaptic transmission in the CA3 area of the hippocampus. J Neurosci 21: 75-83.

Wolters A, Sandbrink F, Schlottmann A, Kunesch E, Stefan K, Cohen LG et al (2003). A temporally asymmetric Hebbian rule governing plasticity in the human motor cortex. $J$ Neurophysiol 89: 2339-2345.

Yamazaki Y, Jia Y, Hamaue N, Sumikawa K (2005). Nicotine-induced switch in the nicotinic cholinergic mechanisms of facilitation of long-term potentiation induction. Eur J Neurosci 22: 845-860.

Supplementary Information accompanies the paper on the Neuropsychopharmacology website (http://www.nature.com/npp) 